Supporting Information

\title{
Modulation of Photochemical Activity of Titania Nanosheets via Heteroassembly with Reduced Graphene Oxide. Enhancement of Photoinduced Hydrophilic Conversion Properties
}

Nobuyuki Sakai, ${ }^{\dagger}$ Kei Kamanaka, ${ }^{\dagger, \downarrow}$ and Takayoshi Sasaki ${ }^{\dagger, \ldots, *}$

${ }^{\dagger}$ International Center for Materials Nanoarchitectonics, National Institute for Materials Science, 1-1 Namiki, Tsukuba, Ibaraki 305-0044, Japan

* Graduate School of Pure and Applied Sciences, University of Tsukuba, 1-1-1 Tennodai, Tsukuba, Ibaraki 305-8571, Japan

*Corresponding author. E-mail: sasaki.takayoshi@nims.go.jp 


\section{Supplementary information}
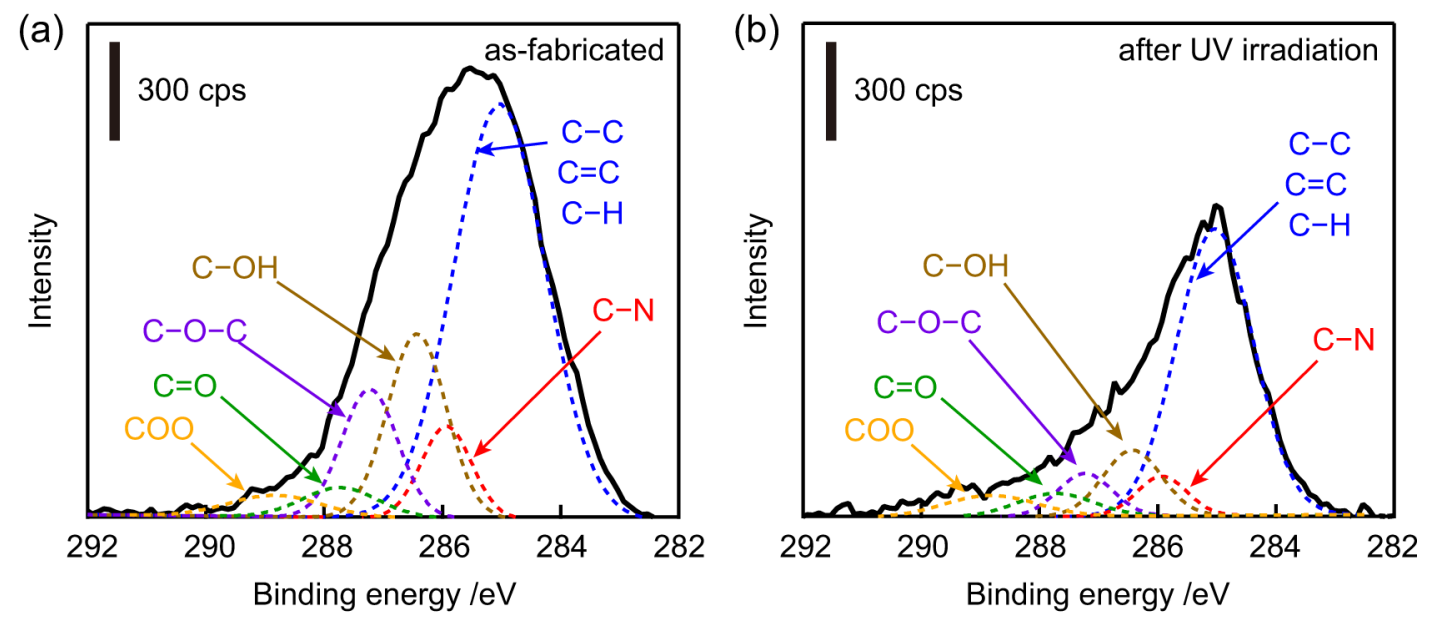

Figure S1. X-ray photoelectron spectroscopy (XPS) data for $\mathrm{C} 1 \mathrm{~s}$ peak for the film of (PDDA/GO/PDDA/Ti $\left.{ }_{0.87} \mathrm{O}_{2}{ }^{0.52-}\right)_{5}$ (a) before and (b) after UV irradiation for $48 \mathrm{~h}$. An XPS system (Theta Probe, Thermo Scientific) was employed to record the spectra.
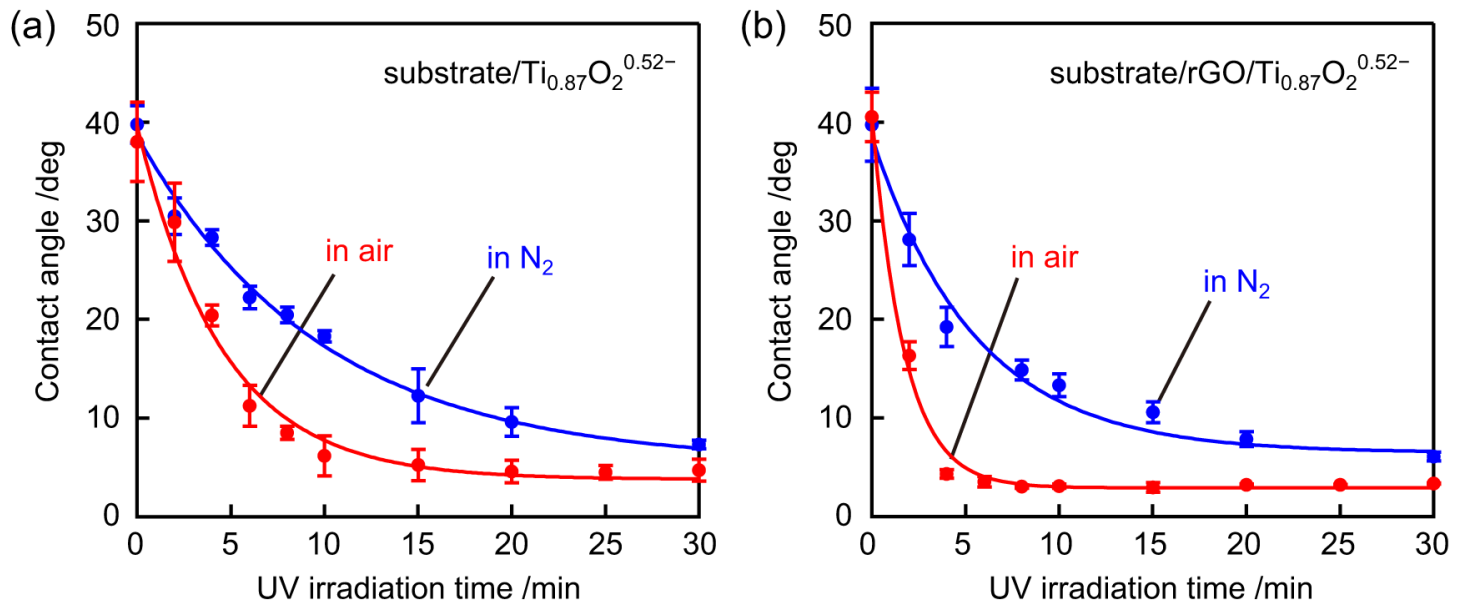

Figure S2. Changes in contact angle for (a) substrate/ $\mathrm{Ti}_{0.87} \mathrm{O}_{2}{ }^{0.52-}$ and (b) substrate $/ \mathrm{rGO} / \mathrm{Ti}_{0.87} \mathrm{O}_{2}{ }^{0.52-}$ under UV irradiation $\left(30 \mathrm{~mW} / \mathrm{cm}^{2}\right)$ in ambient air (red circles) or a humid $\mathrm{N}_{2}$ atmosphere (blue circles). 